\title{
5. HARD COSMIC X-RAY SOURCES
}

\author{
L. E. PETERSON \\ Dept. of Physics, University of California, San Diego, La Jolla, Calif. 92037, U.S.A.
}

\begin{abstract}
A review of the observational status of X-ray sources detected in the $20 \simeq 500 \mathrm{keV}$ range is presented. Of the approximately 115 sources listed in the March 1972 edition of the UHURU 2-6 keV sky survey catalog, about 15 sources have been studied in hard X-rays. Most of the data have been obtained from balloons, although the OSO-3, and more recently the OSO-7, have contributed. With the exception of CEN A, the SMC, and possibly M-87, all the sources detected at higher energies are galactic and heavily concentrated in the galactic plane. The Crab Nebula has been measured to about $500 \mathrm{keV}$ in continuous emission and a component at the $\simeq 33 \mathrm{~ms}$ pulsar period comprising about $20 \%$ of the total emission has been detected to $\sim 10 \mathrm{MeV}$. Objects such as SCO-1 and CYG-2 are characterized by an exponential spectrum, which varies over a $10 \mathrm{~min}$. time scale about a factor of two, and a flatter spectrum extending to above $40 \mathrm{keV}$ which exhibits independent variability. Objects such as CYG-1 and possibly CYG-3 have a multi-component power law spectrum extending to over $100 \mathrm{keV}$, and may vary many factors over a period of weeks. Other sources generally not yet identified with optical or radio candidates, located in the Galactic Center and the Centaurus/Crux region also show considerable variability, and in one case may have been detected to nearly $500 \mathrm{keV}$. Only upper limits at about $2 \times 10^{-4}$ photon $\left(\mathrm{cm}^{2} \mathrm{~s} \mathrm{keV}\right)^{-1}$ in the $20-50 \mathrm{keV}$ range exist for most supernova remnants and extragalactic sources.
\end{abstract}

\section{Introduction}

This is a brief review of the observational knowledge of cosmic X-ray sources in the twenty to several hundred $\mathrm{keV}$ range. This work is not intended to be complete, but will summarize recent results, indicate future directions, and update a similar review given at a preceeding IAU Symposium (Peterson, 1970). We will also include data obtained by UCSD on the OSO-7 satellite and on recent balloon flights which have not yet appeared in the open literature. In particular, observations of hard X-rays will be interpreted in terms of the catalog of sources generated from the UHURU data (Giaconni et al., 1972).

The majority of X-ray emitters observed by rockets and by the UHURU satellite have a soft spectrum, therefore, the relative sensitivity must be increased at higher energies to detect the same number of sources. Since sensitivity is a more difficult problem at these energies (Peterson et al., 1971), the number of observed sources is less. Of the 115 sources listed in the UHURU 2-6 keV catalog, only about 20 have been detected above $15 \mathrm{keV}$. The sensitivity level with detectors available at the present time is in the range of 1 to $2 \times 10^{-4}$ photons $\left(\mathrm{cm}^{2} \mathrm{~s} \mathrm{keV}\right)^{-1}$ in the $20-50 \mathrm{keV}$ range. The most useful work in this range has been obtained by extending the spectrum, which must be a signature of the radiating mechanism, and studying the time variability which relates to models based on compact sources or binary configurations. Discovery and identification is best accomplished at lower energies.

Only two objects, the Crab Nebula (Peterson and Jacobson, 1970) and a source near the Galactic Center (Johnson et al., 1972) have a measured spectrum extending significantly beyond $300 \mathrm{keV}$. Several cosmic sources, GX 1+4, GX 304-1, and 
GX 301-2, all of which are variable, have been discovered first from balloon observations. All confirmed measurements thus far have been limited to continuum type of spectra, although $\gamma$-ray lines have been searched for to a sensitivity level of about $10^{-3}$ photons $\left(\mathrm{cm}^{2} \mathrm{~s}\right)^{-1}$, and Haymes (Johnson et al., 1972) has reported a barely significant measurement of a line near $0.511 \mathrm{MeV}$ from the Galactic Center. Two extra-galactic sources, CEN A or NGC 5128 (Lampton et al., 1972) and the Small Magellanic Cloud (Price et al., 1971) has been positively detected at energies greater than $20 \mathrm{keV}$. A number of results have also appeared on the Virgo Cluster, or possibly $\mathrm{M} 87$, which indicate a detectable spectrum beyond $50 \mathrm{keV}$.

\section{Instrumentation}

Before discussing measurements of various sources and their physical significance, it is useful to indicate some concepts of detection technique. Detectors used in the 20$500 \mathrm{keV}$ range usually consist of scintillation counters with active or semiactive anticoincidence collimators (Peterson, 1970; Peterson et al., 1971), although Glass (1969) has used proportional counters to obtain very large areas. Scintillation telescopes have thus far been less than $\dot{a}$ few hundred $\mathrm{cm}^{2}$ with apertures not smaller than a few degrees. Table I indicates instruments used to obtain certain significant observations in the $>20 \mathrm{keV}$ range, and includes reference citations in this paper.
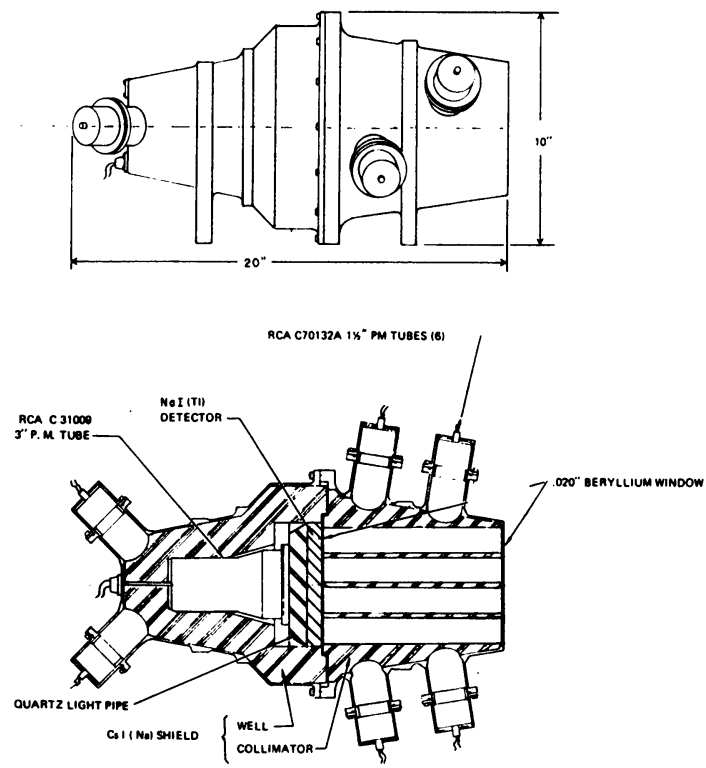

OSO. 7 COSMIC X. RAY TELESCOPE
UNIVERSITY OF CALIFORNIA. SAN DIEGO

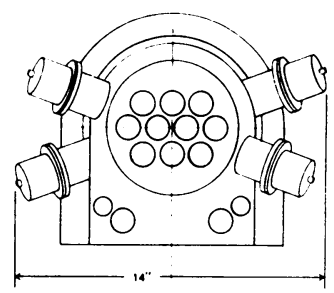

SPECIFICATIONS DETECTOR

SENSITIVE AREA

THICKNESS

FIELD OF VIEW (FWHM)

SOLID ANGLE

$$
\text { SHELD }
$$

ISOTROPIC GEOMETRY FACTOR TOTAL DETECTOR WT.

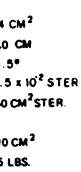

$\mathrm{cm}^{2}$

$1.5 \times 10^{-2}$ STEA

$610 \mathrm{~cm}^{2}$

L LBS

Fig. 1. The telescope used by UCSD on the OSO-7 satellite consists of a combination of scintillation counter elements providing detection and active anti-coincidence collimation features. This detector is similar to those used by others in the $20-500 \mathrm{keV}$ energy range. 
HARD COSMIC X-RAY SOURCES

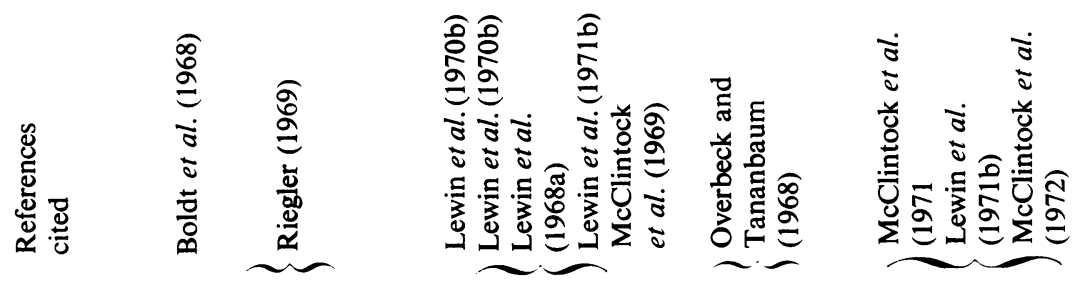

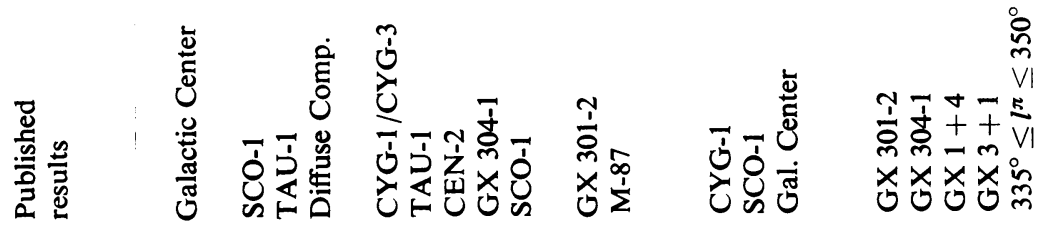

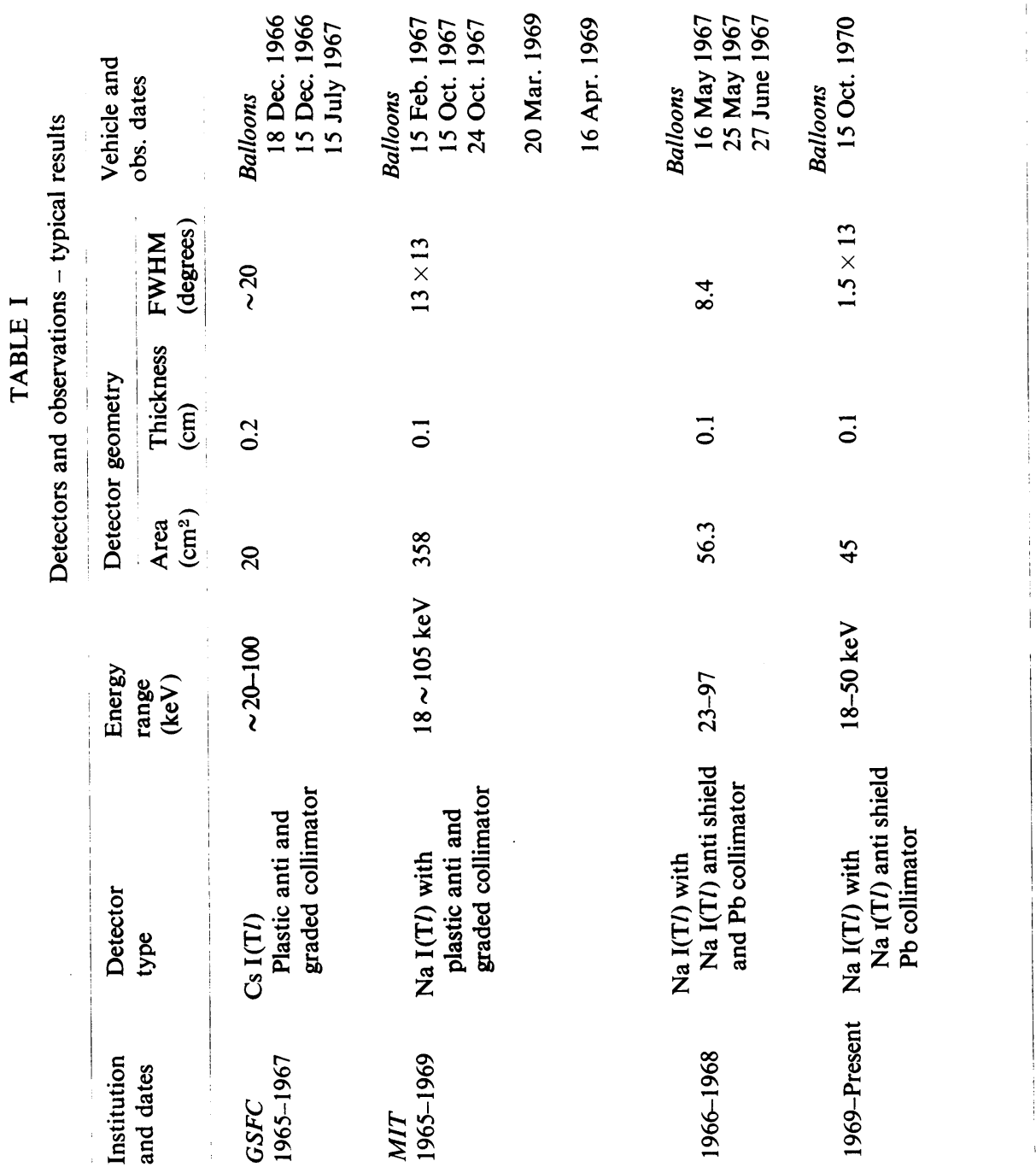




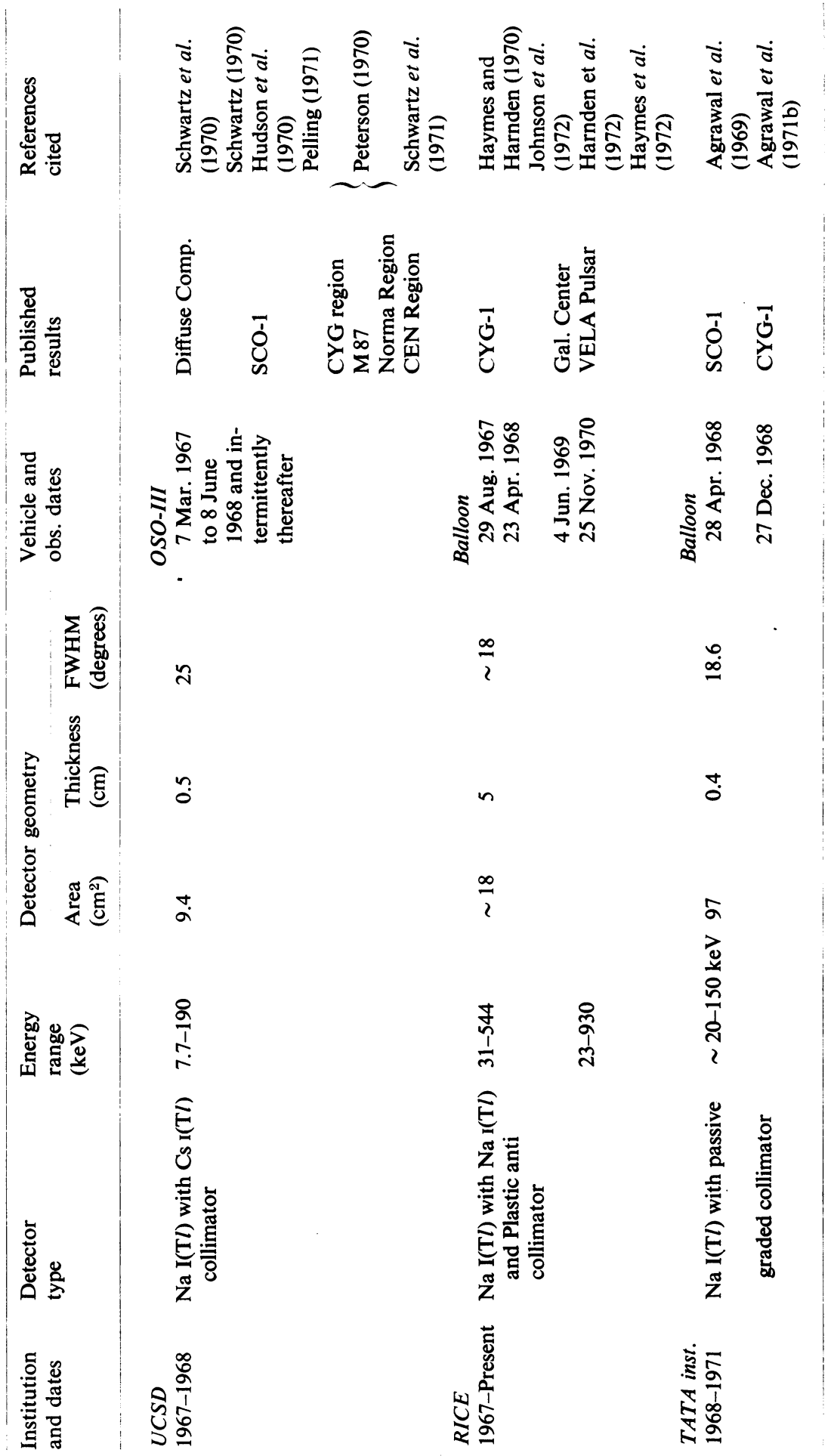




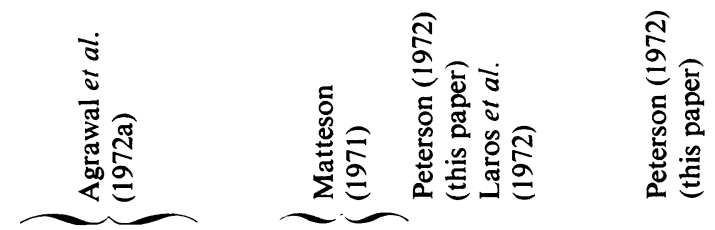

m

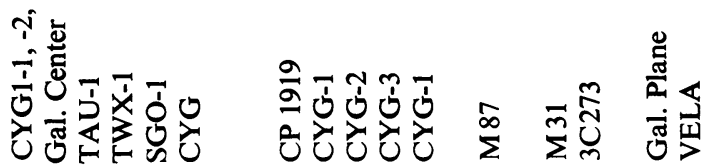

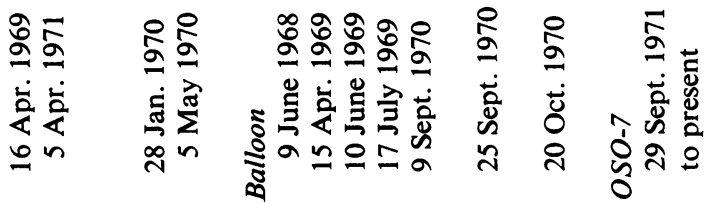

$\stackrel{0}{i} \quad \dot{m}$

?

$\stackrel{+}{\circ} \stackrel{\circ}{-}$

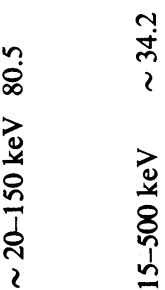

t

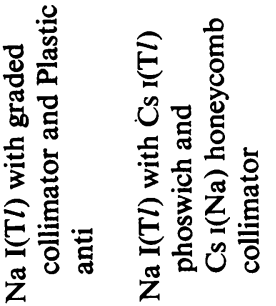

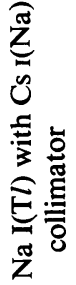

$\frac{\sqrt{2}}{\sqrt[3]{8}}$

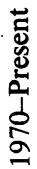

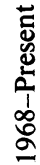

ลิ 
Most observations have been obtained from balloons or satellites because of the short exposure during a rocket flight. Although atmospheric cut off at depths of 2-3 $\mathrm{gm} / \mathrm{cm}^{2}$ usually limits measurements to above $20 \mathrm{keV}$, MIT (McClintock et al., 1972) has succeeded in using exceedingly large balloons to carry instruments to a depth less than $1.5 \mathrm{gm} / \mathrm{cm}^{2}$. Recently, significant satellite observations have been obtained by UCSD on OSO-3 (Schwartz et al., 1970; Schwartz, 1970), by Frost (1969) on the OSO-5 and Brini on the OSO-6. The UCSD experiment on OSO-7 will add further knowledge on cosmic X-ray sources.

The telescope configuration on the OSO-7, shown in Figure 1, is described here since it is also typical of those used recently on balloons. The sensitive element consists of a $1 \mathrm{~cm}$ thick $\mathrm{Na} \mathrm{I}(\mathrm{Tl})$ crystal inside a massive $\mathrm{Cs} \mathrm{I}(\mathrm{Na})$ anticoincidence shield. Holes drilled in the Cs $\mathrm{I}(\mathrm{Na})$ collimator define the aperture. This detector has an effective area of $64 \mathrm{~cm}^{2}$, a FWHM response of $6.5^{\circ}$, and operates over the $7.0-550 \mathrm{keV}$ range. Instruments located in the rotating wheel of an OSO usually point radially outward, and therefore scan great circles with the nominal two-second rotation period. In the UCSD instrument, each event is analyzed into 128 energy-loss channels and transmitted as an event signature which also contains satellite clock time to $0.625 \mathrm{~ms}$. This time may be used to obtain the X-ray direction, knowing the instantaneous satellite rotation vector, or as event timing for pulsar analysis.

\section{Source Location and Identification}

Here we discuss hard X-ray sources by regions on the celestial sphere, indicating relative source strengths, variability, and identification. In particular, we attempt to identify sources measured from balloon or satellite work with entries in the UHURU catalog (Giacconi et al., 1972). Source identification is difficult because of variability in strength and spectral shape, and because of confusion due to close spacing.

\section{A. GALACTIC PLANE SCAN}

Here we present preliminary results obtained from the UCSD instrument on the OSO7, which was launched September 29, 1971. These results were obtalned in December 1971 when the OSO-7 was scanning along the galactic equator, and represent about five 90 -min orbits of quick-look data for an exposure per source of about $50 \mathrm{~s}$. The sources, locations and scan range are shown on the map in Figure 2, where preUHURU source names are used. The counting rate as a function of galactic longitude is shown in Figure 3 for the energy ranges $7.0-26 \mathrm{keV}$ and $>26 \mathrm{keV}$. Also shown is an equivalent UHURU scan (Giacconi et al., 1971) obtained approximately one year earlier. Sources which appear consistently in both UHURU and OSO scans are indicated.

The most striking feature is the softness of the X-ray sources. Although the 7-26 $\mathrm{keV}$ range shows nearly as many sources as in the UHURU scan, the $>26 \mathrm{keV}$ shows only two sources, CYG-1 and the Crab Nebula. The OSO-7 scan does not show the Galactic Center on this date since it coincided with the Sun, and a solar cell inhibits 


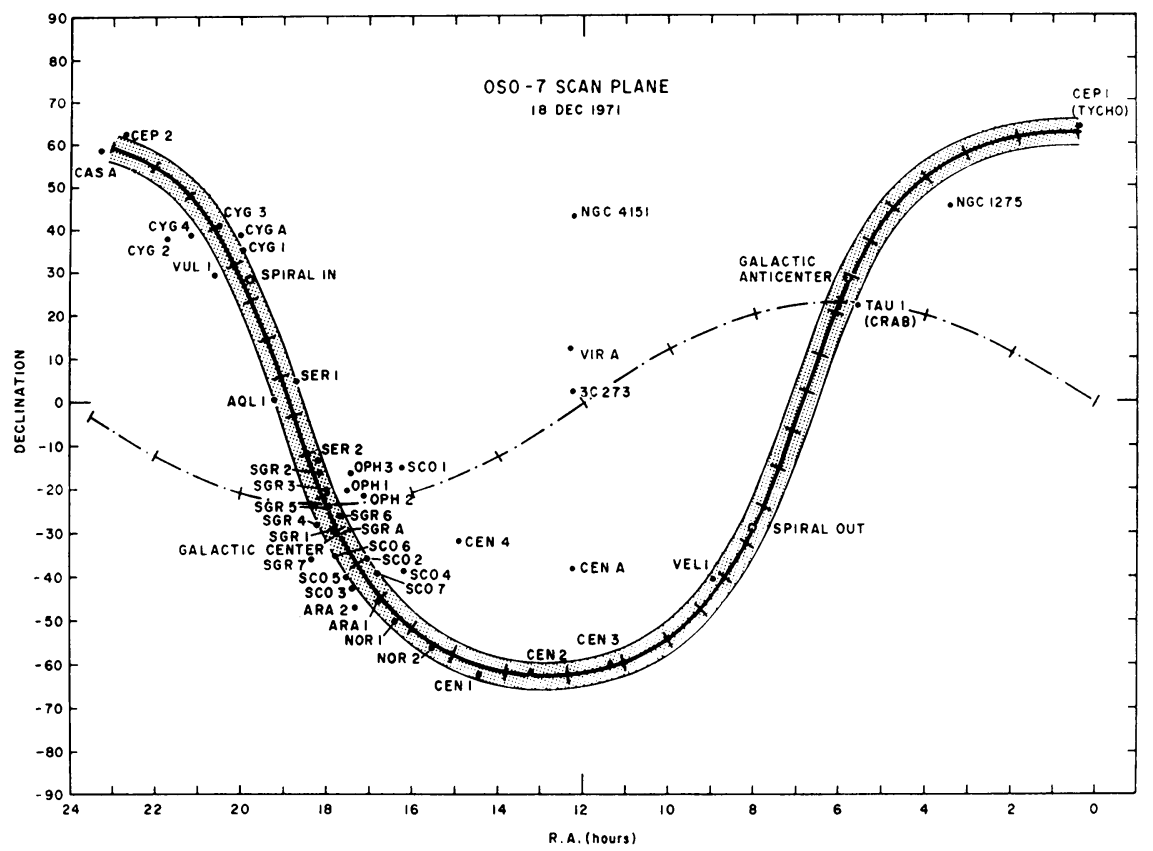

Fig. 2. This map of the celestial sphere shows most of the X-ray sources detected previous to the UHURU. The scan path along the galactic plane of the OSO-7 on 18 Decenber 1971 is also shown.

readout to prevent excessive telemetry deadtime due to solar X-rays. No correction in either of these scans has been made for the aperture response; in particular, the Crab Nebula was nearly out of the OSO-7 aperture. It is not immediately clear that differences between UHURU and 7-26 keV OSO-7 scans occur because of a soft spectrum, the aperture response, or are due to time variations in the one year interval.

The relatively strong source $2 \mathrm{U} 0613+09$, at approximately $200^{\circ}$ galactic longitude was not observed in the OSO-7 data. The source VEL-1 (2U0900-40) is observable in the OSO-7 data, and additional results on the Vela Region will be indicated later in this paper. The pulsating source CEN-3 (Schreier et al., 1972) apparently has a significantly softer energy spectrum than the Crab Nebula. CEN-2, the first source observed to have a nova-like behavior, is apparently not indicated in either the UHURU catalog or the OSO data. The source as identified CEN-1 in Figure 3 may actually be GX 304-1, which was discovered by Lewin et al. (1971a) and is indicated as 2U1258-61 in the AS \& E catalog. Furthermore, the OSO-7 data clearly indicates more than one source in this region. The sources in the Lupus/Norma complex are not also well resolved in the OSO results, however, the rather strong source CIR X-1 at $l^{\mathrm{II}}=322^{\circ}$ (2U1516-56) either has an exceedingly soft spectrum or was unobservable one year later in hard X-rays. In general, sources in the Lupus/Norma region have been reported as being variable both by the UHURU and by balloon (McClintock et al., 1972) and satellite results (Schwartz et al., 1971). The source ARA-1 (2U1641-45 ?) reported as 


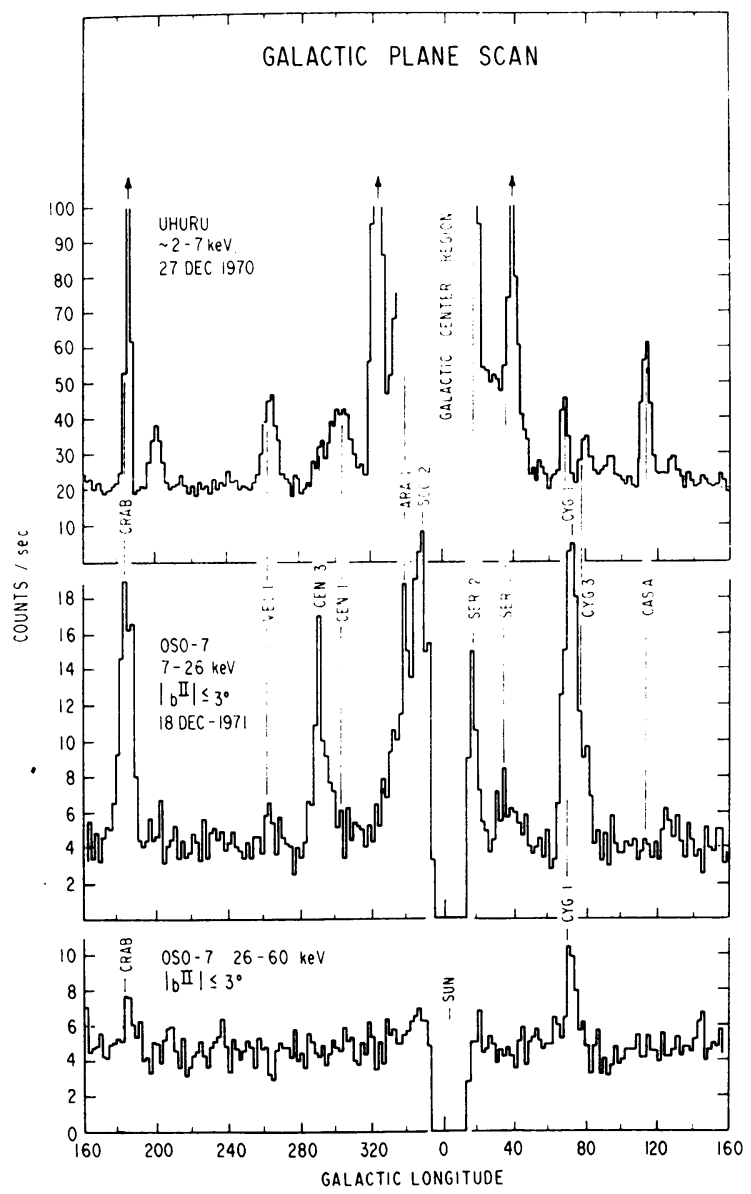

Fig. 3. Sources identified in the OSO-7 galactic plane scan compared with those seen at lower energies one year earlier by the UHURU. An obvious feature is the softness of the typical X-ray source spectrum.

variable in the UHURU catalog, is apparently soft, although it may be confused with other strong variable sources within a few degrees galactic longitude. A source within $20^{\circ}$ of the Galactic Center, possibly SCO-2 or SCO-6, may just show in the $>26 \mathrm{keV}$ data. Galactic Center sources, as already indicated, háve been gated out by the OSO-7; they will be discussed in a later section.

Northward from the Galactic Center, SER-2 (2U1813-14) also shows strongly. The strong source identified as SER-1 was nearly out of the aperture, and therefore the structure near $l^{\mathrm{II}} \cong 30^{\circ}$ may be associated with other sources in this region. CYG-1 and CYG-3 are clearly resolved in the 7-26 keV range, however, at the higher energies only CYG-1 is well above background. According to Gorenstein et al. (1970), the supernova remnant CAS A has a steep spectrum, and may therefore not be expected to show in the OSO-7 data. 


\section{B. GALACTIC CENTER REGION}

Earlier observations of the Galactic Center are somewhat confused by the wide aperture telescopes (Peterson, 1970; Boldt et al., 1968; Riegler, 1969; Agrawal et al., 1971a; Overbeck and Tananbaum, 1968) and the apparent high variability of the sources. Recent observations by Lewin et al. (1971a) over the $18-50 \mathrm{keV}$ range have indicated the presence of a strong source GX $1+4$, and possible fluxes from GX $3+1$ and GX 5-1. The spectrum of GX 9+9 has also been measured by the Tata Institute (Agrawal et al., 1971a). Sources at these locations are identified in the UHURU catalog, and most have also been indicated in earlier rocket surveys by MIT (Bradt et al., 1971), as shown in Figure 4. Although possibly not completely resolved, GX $1+4$ is



Fig. 4. Source locations in the galactic center region as identified by MIT and UHURU surveys. Only the source GX $1+4$, and possibly GX $3+1$ have been isolated in $>20 \mathrm{keV} \mathrm{X}$-rays, although numerous results obtained with wide angle telescopes on the total region have been reported.

reported by Lewin as having a hard spectrum corresponding to a temperature of $28 \pm 12$ $\mathrm{keV}$, or a power law number spectrum with an index of $-2.4 \pm 0.7$. Agrawal et al. (1971a) report a similar spectrum from the galactic center region, even though the sources were not resolved. Haymes (Johnson et al., 1972) has also measured this region with a wide aperture detector and determined a hard spectrum with a power law index of $2.37 \pm 0.05$ from $23-930 \mathrm{keV}$. Although this was tentatively associated with GX 5-1, most likely it should be identified with GX $1+4$ since the power law index agrees with Lewin. According to the UHURU catalog, there is a diffuse source (2U1743-29) of about $2^{\circ}$ extent associated with the Galactic Center which balloon measurements have not yet been able to resolve. Haymes has also identified a $\gamma$-ray line structure at 
approximately $0.5 \mathrm{MeV}$ with an intensity of $1.8 \pm 0.5 \times 10^{-3}$ photons $\left(\mathrm{cm}^{2} \mathrm{~s}\right)^{-1}$ associated with the Center region. Although there are qualifications to this observation, verification would add a new and exciting element to $\gamma$-ray astronomy.

\section{CEnTAURUS/CRUX}

This region is characterized by outstanding examples of source variability and flaring (Lewin et al., 1971b; McClintock et al., 1971; Schwartz et al., 1971). CEN-2, which was first observed to have a nova-like behavior in soft X-rays in early 1967 (Chodil et al., 1968), was thought to have been observed in a series of balloon flights and by OSO-III in hard X-rays. There is no source identified in the UHURU catalog at the improved galactic longitude of $310.2 \pm 1^{\circ}$ given by Francey (1971). It is not obvious that CEN-1, CEN-4 and CEN-5 were ever observed from balloons.

During 1969 and 1970, Lewin (McClintock et al., 1971) has reported two new sources, GX 304-1 and GX 301-2, both with high variability. The former is certainly identified with 2U1258-61; it had been observed earlier and mistakenly thought to be CEN2 (Lewin et al., 1970b, 1971b). The factor of 7 decrease reported for CEN 2 between October 1967 and March 1969 is probably associated with GX 304-1. GX 301-2 is identified with 2U-1223-62. Figure 5, adapted from Schwartz et al. (1971) shows the location of sources in the Centaurus region, compared with the UHURU catalog. CEN-1, CEN-2 and CEN-5 apparently have not been verified by the UHURU. Little has been published on the spectra of these sources at higher energies, although some lower energy data are available (Hill et al., 1972).

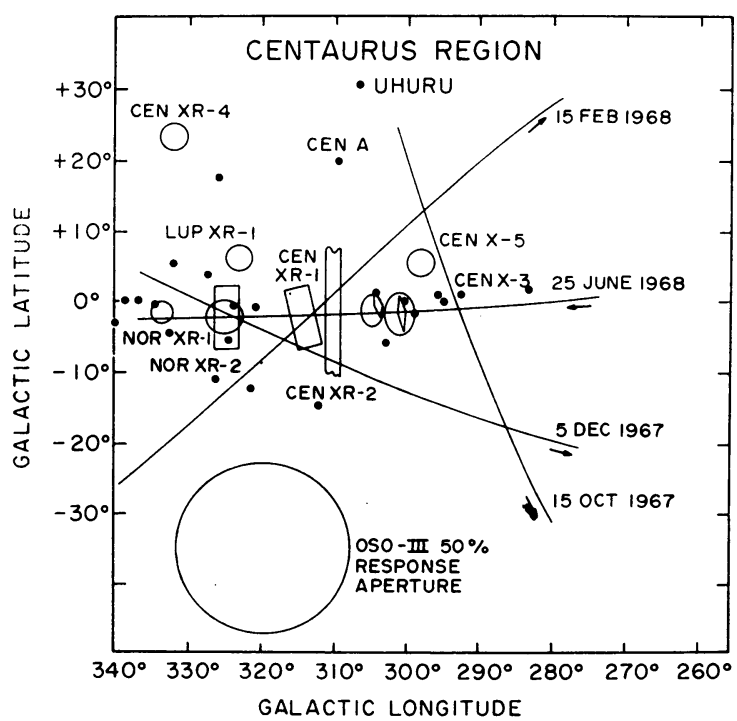

Fig. 5. The Centaurus region showing known X-ray sources. The ellipses and diamonds are variable sources observed by the MIT balloon group. The scan lines are those of the OSO-III. The sources GX 304-1 and GX 301-2 have been observed to exhibit considerable variability in hard X-rays. The redetermined position of CEN-2, shown here, implies hard X-rays earlier associated with it are due to other sources. 
CEN 3, the remarkable modulated pulsating source (Schreier et al., 1972) believed associated with a rotating neutron star as a binary companion to a larger star, has not yet been observed from balloons, although it is evident from the OSO-7 data that the spectrum cuts off rather steeply above $26 \mathrm{keV}$.

\section{CYGNUS SOURCES}

Early balloon and satellite observations failed to resolve the Cygnus sources so that interpretation in terms of variability and spectrum may be unclear. CYG-1, assumed to be the strongest source in this region has a hard spectrum (Haymes and Harnden, 1970; Webber and Reinert, 1970) and has been observed many times from balloons. In particular, the work of Overbeck and Tananbaum (1968) showed this source to be highly variable in hard X-rays. These sources are resolved in more recent observations, so unambiguous knowledge of their spectrum and variability is now available.

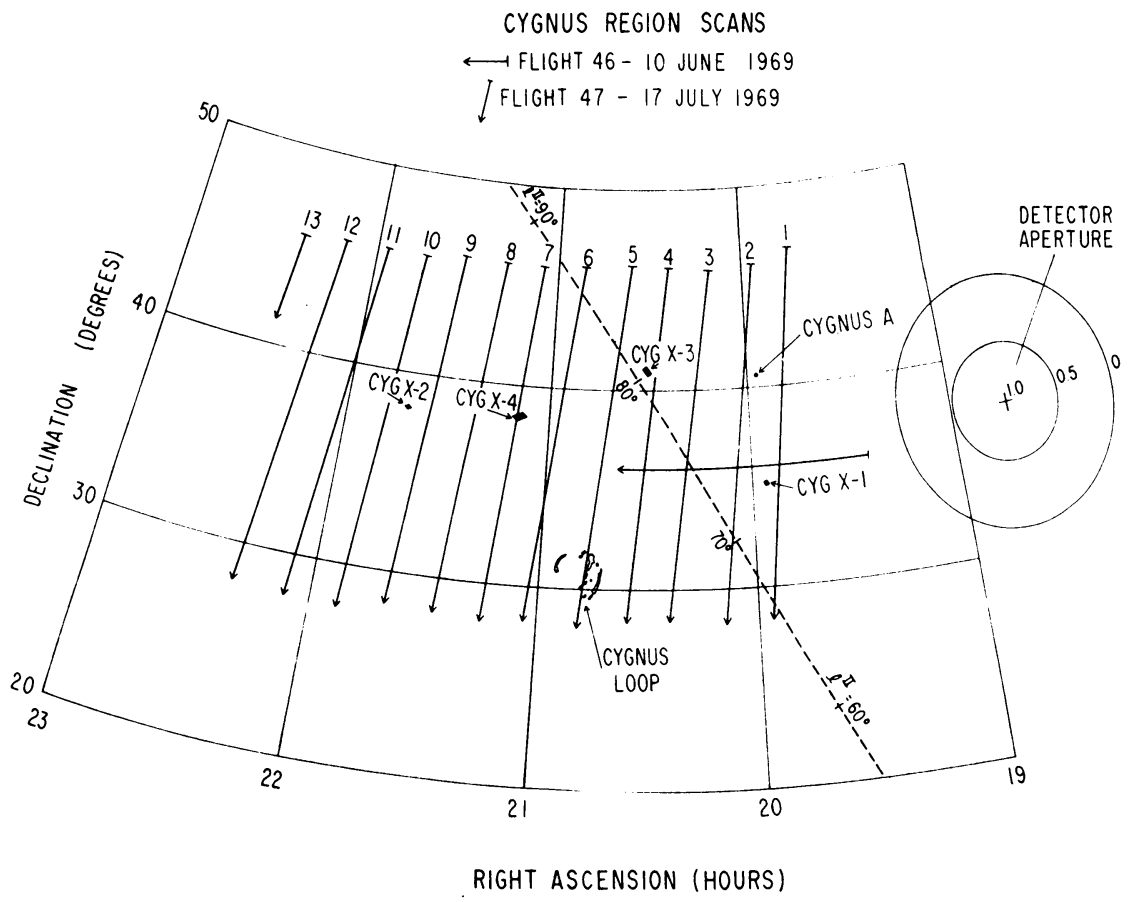

Fig. 6. This shows the location of identified X-ray sources and other interesting objects in the Cygnus region. The scan paths are those of Matteson during his high resolution balloon survey of the region. Only CYG-1, -2 , and -3 have been detected $>20 \mathrm{keV}$.

Figure 6, taken from the thesis of Matteson (1971) shows the location of the principal sources, CYG-1, CYG-2 and CYG-3. Apparently, CYG-4 has never been observed from balloons; furthermore, it is not indicated in the UHURU catalog. Only upper limits exist for the Cygnus Loop and the radio source CYG A (Matteson, 1971). 


\section{E. SUMMARY}

From the spatial distribution of hard X-ray sources, inferences can be made about the nature of the emitting objects. Except for a paucity of sources in Orion and in the Galactic anti-center direction, these tend to cluster in a manner which delineates spiral arms. These sources are therefore probably not of extreme Pop. I, but may be old Pop. I, and perhaps even some Pop. II objects distributed toward the Galactic center. This suggests X-ray 'stars' are not associated with the formation of normal stars, but are a part of stellar evolution not yet understood. The spatial distribution of similar X-ray emitting objects provides information relative to their place in stellar evolution and their distance. At present, these sources are not well enough understood to permit these investigations.

\section{Source Types}

This is a more complete discussion of the various sources in terms of their characteristic spectra and the type of objects with which they have been identified.

\section{A. THE CRAB NEBULA}

Observations on this unique source have been summarized by Peterson and Jacobson (1970). The number spectrum is represented as a power law with an index of -2.25 from about $1-500 \mathrm{keV}$, as shown in Figure 7. Long term time variations have not been noted. Following the discovery in 1969 of the $33 \mathrm{~ms}$ radio pulsar located near the center of the nebula, optical and X-ray pulsed emission were soon measured. Pulsed X-ray measurements now extend to $3.5 \mathrm{MeV}$, (Kurfess, 1971; Orwig, et al., 1971), and account for at least $20 \%$ of the total flux as shown in Figure 7. A compilation of data on the pulsed and steady components (Figure 1 of Fazio's review, this volume p. 303) shows that at higher energies an increasing fraction of the energy is pulsed.

The steady emission presumably has its origin within the $1^{\prime}$ or $2^{\prime}$ size identified by rocket observations with the amorphous nebulosity. No information is yet available on the source size as a function of energy. Since the synchroton process is thought to be responsible for the continuous component, such measurements would provide information regarding the diffusion and life-time of energetic electrons in the nebula itself. Observations to detect $\gamma$-ray line emission have been performed, but only upper limits at an intensity of about $10^{-3}$ photons $\left(\mathrm{cm}^{2} \mathrm{~s}\right)^{-1}$ are available (Peterson and Jacobson, 1970). Based on the $\mathrm{Cf}^{254}$ hypothesis for the explanation of the 55 daylight curve of type I supernovae, intensities of about $3 \times 10^{-4}$ photon $\left(\mathrm{cm}^{2} \mathrm{~s}\right)^{-1}$ are expected for $\gamma$-rays due to $\mathrm{Am}^{241}$ at $62 \mathrm{keV}, \mathrm{Cf}^{251}$ at $180 \mathrm{keV}$ and $\mathrm{Cf}^{249}$ at $390 \mathrm{keV}$. Although the status of observational data on the $\mathrm{Cf}^{254}$ hypothesis has not changed since last reviewed, the entire idea seems less likely in view of the association of the central pulsar with a collapsed star.

\section{B. SUPERNOVA REMNANTS}

No other observed X-ray source or supernova remnant has all the characteristics of 




Fig. 7. The spectrum of the Crab nebula determined between 1 and $500 \mathrm{keV}$, assembled from rocket and balloon results. Only certain selected data are shown here, though all the published data were taken into account (see Gorenstein et al., 1970, and Pounds [this volume] for references). The power law spectrum is thought to be indicative of the synchroton process. About $20 \%$ of the emission in this spectrum is pulsed.

the Crab Nebula with the possible exception of VELA X; other supernova remnants have not yet been observed above $10 \mathrm{keV}$ despite many attempts. Such objects, observed in the 2-10 keV range (Gorenstein et al., 1970) have a spectrum characteristic of a hot gas not exceeding 10 or $20 \times 10^{6} \mathrm{~K}$ and a relatively low luminosity of about $10^{36}$ ergs s$^{-1}$. In particular, CAS A, Tycho, and the Cygnus Loop (Matteson, 1971) have limits $\sim 6 \times 10^{-4}$ photons $\left(\mathrm{cm}^{2} \mathrm{~s} \mathrm{keV}\right)^{-1}$ in the $20-50 \mathrm{keV}$ range. A hard X-ray component, if it exists in these objects, must have a luminosity less than a few percent of the total.

Figure 8 shows the Vela region which contains the Vela supernova remnant, radio pulsar PSR 0833-45, and an UHURU source identified with this object. Harnden et al. (1972) have reported a source in this region, and have found marginal evidence for pulsed X-ray emission in the $23-80 \mathrm{keV}$ range with a period $150 \mathrm{~ns}$ less than the 


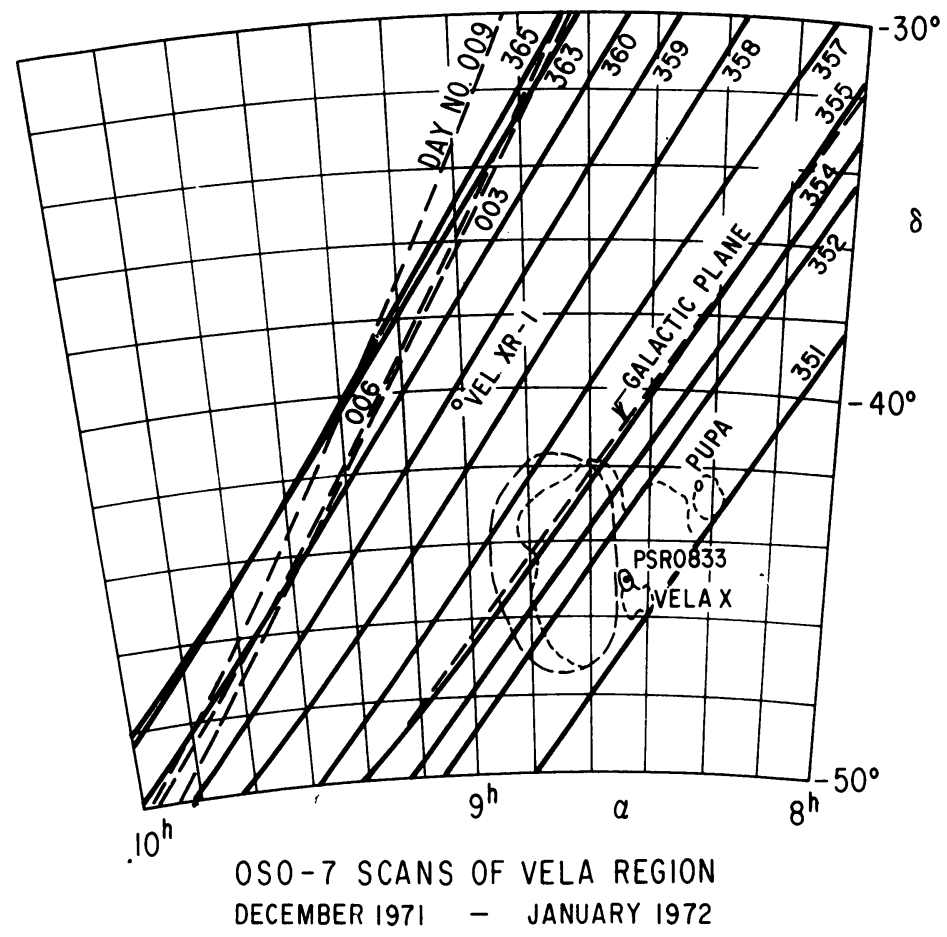

Fig. 8. This shows locations of the X-ray source VEL-1, and objects associated with the Vela $X$ supernova remnant, together with scan paths of the OSO-7 X-ray instrument in

December 1971/January 1972.

nominal $89^{+}$ms. Except for the pulsed feature, this emission could be associated with the variable X-ray source VEL-1. The OSO-7 scanned this region from December 17, 1971 through January 10, 1972 and failed to detect X-rays from the location of VEL X at a $2 \sigma$ limit of $4 \times 10^{-2} \mathrm{keV}\left(\mathrm{cm}^{2} \mathrm{~s} \mathrm{keV}\right)^{-1}$ in the 7-26 keV range.

\section{SCO-1 TYPE SOURCES}

SCO-1 is characterized by an exponential spectrum whose equivalent temperature is on the order of $50 \times 10^{6} \mathrm{~K}$ and is identified with a flickering blue star-like object. The $\mathrm{X}$-ray spectrum in the $1-10 \mathrm{keV}$ range varies a factor of 2 , apparently at random, although a recent analysis (Angel et al., 1971) has indicated oscillation in the $1-10 \mathrm{~Hz}$ range persisting for a minute. Early observations indicated the spectrum above $40 \mathrm{keV}$ also contained an additional variable compohent. This has now been verified to about $120 \mathrm{keV}$ (Agrawal et al., 1969; Lewin et al., 1970a ; Haymes et al., 1972). Simultaneous optical observations (Toor et al., 1970; Pelling, 1971) have shown a coupling to the lower energy X-rays, and therefore indicated a common region. Radio and optical variations show no such correlation (Lampton et al., 1971). Soft X-ray and optical observations may be interpreted in terms of a hot gas model which becomes optically thick in the UV. The hard X-ray and radio fluxes are inconsistent with this 
simple model and may require an additional source region. Perhaps simultaneous observations of these components are also appropriate, since both have demonstrated time variability.

In addition to the random variations, SCO-1 also exhibits a flare-like phenomenon which extends into the hard X-ray region (Lewin et al., 1968a) and is associated with an optical increase (Hudson et al., 1970; Pelling, 1971). The bursts have about a tenminute duration, a factor of two increase in X-ray intensity and a spectrum similar to that of the quiescent state.

Because of its intensity, SCO-1 has been the most studied source of this type. However, CYG-2 has also been tentatively identified with a star-like flickering object (Kraft and Demoulin, 1967). The compilation of data shown in Figure 9 taken from Matteson's thesis (1971) indicates the exponential spectrum believed associated with the $50 \times 10^{6} \mathrm{~K}$ gas and the flat, high energy component. Both features of the spectrum in this source may also show variability.

D. CYG-1 TYPE SOURCES

CYG-1 is characterized by a spectrum which extends to several hundred keV (Haymes and Harnden, 1970); rapid, possibly quasi-periodic variations in soft X-rays (Terrell,

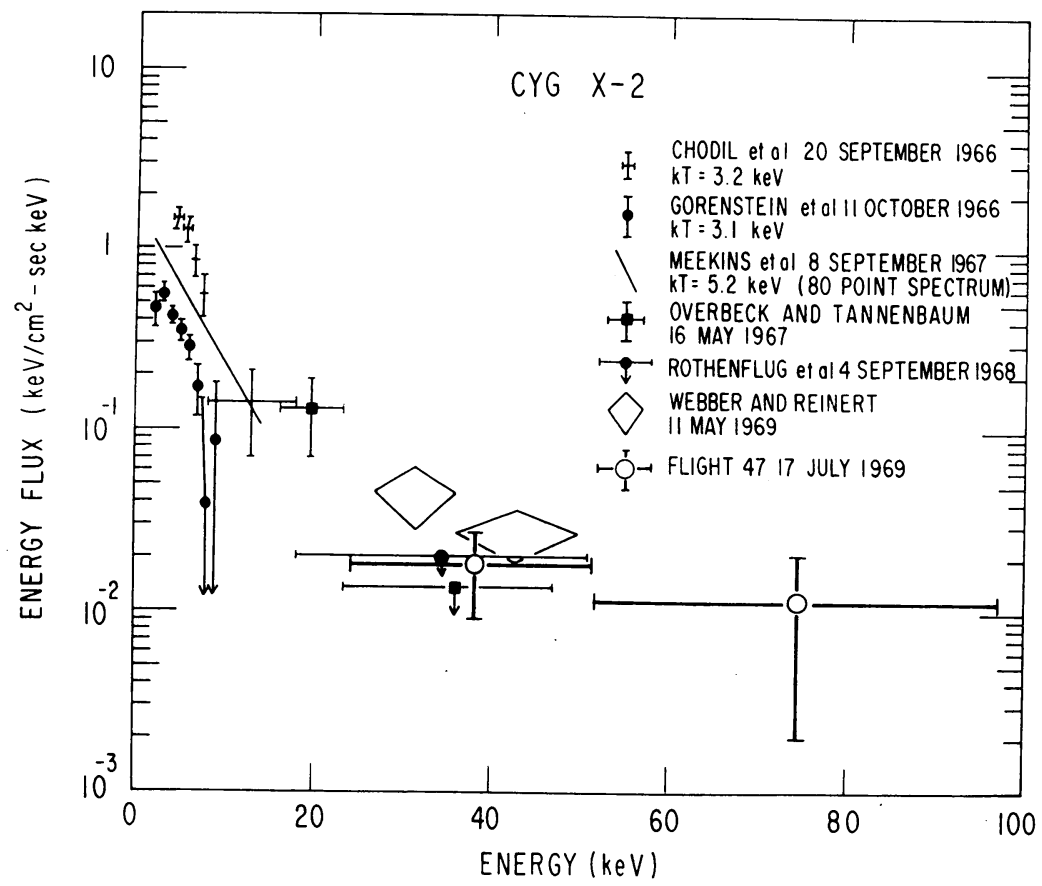

Fig. 9. Spectral data on CYG-2, taken from Matteson's thesis (1971), where the references may be found. At energies below $15 \mathrm{keV}$ a thermal spectrum with factor two intensity and temperature variations is observed, while higher energies show a hard spectral component which is also variable. A similar high energy feature is seen in the spectrum of SCO-1. 
1972); and large changes in hard X-ray intensity (Overbeck and Tananbaum, 1968). Recent observations have indicated that the hard X-ray spectrum of CYG-1 is characterized by 'high' and 'low' states. Figure 10 shows the results obtained by Matteson (1971) together with other spectral data. A flight on 10 June 1969 indicated a hard

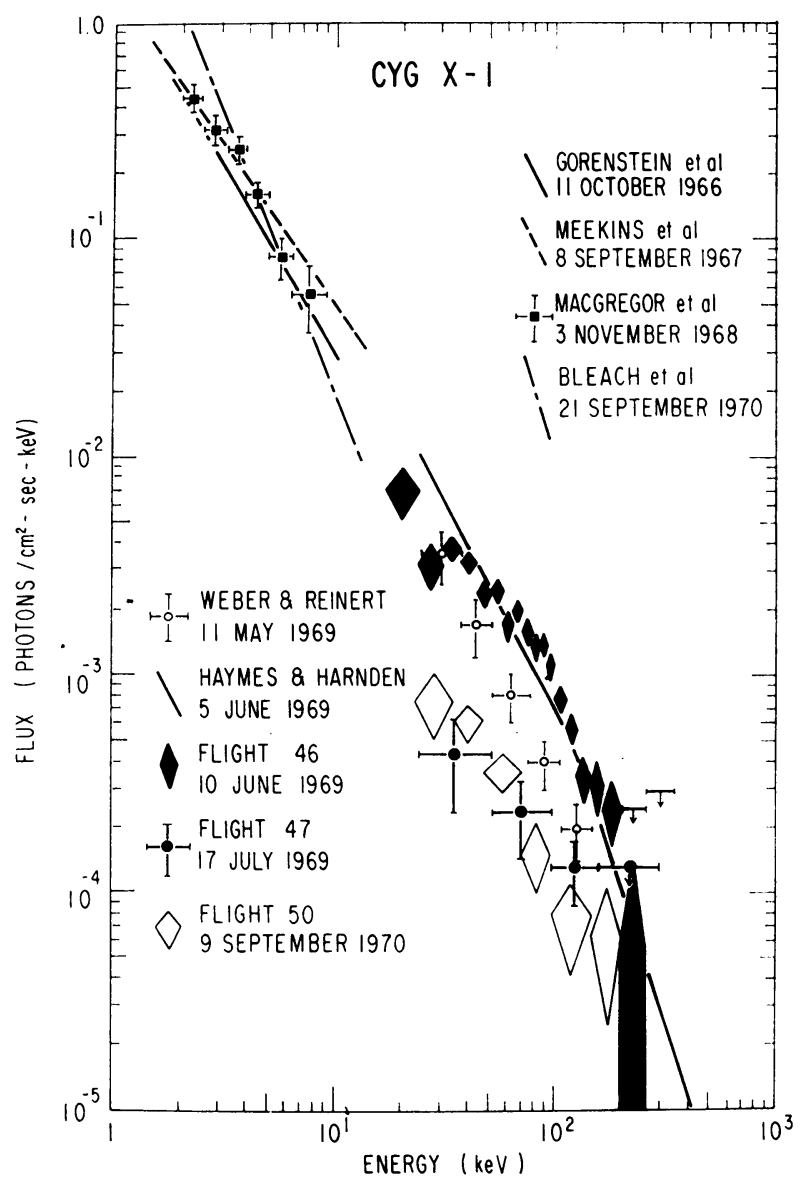

Fig. 10. A compilation of rocket and balloon spectral data on CYG-1, taken from Matteson (1971). During a series of balloon observations, May to June 1969, large variations in intensity and complex spectral changes occurred at energies above $20 \mathrm{keV}$. The intensity on 17 July 1969 is the lowest ever measured from CYG-1 at energies above $20 \mathrm{keV}$.

spectrum, in agreement with other observations which were made during the preceeding month. Less than one month later, an additional observation found CYG-1 in a much weaker state, as did an observation by USCD about a year later. Reference citations shown in Figures 9, 10, and 11, may be obtained from Matteson (1971).

The source has now been tentatively identified with a 5.6 day binary (Bolton, 1972; Webster and Murdin, 1972). Unlike CEN-3, 5.6 day variations possibly associated 
with an occultation of the X-ray emitting neutron star by large central star have not been observed in the soft X-ray region by UHURU. The OSO-7 observed CYG-1 between 16 and 22 December 1971 when the source was generally in a 'high' state. Although large changes in hard X-rays were observed, no turn-off occurred at the

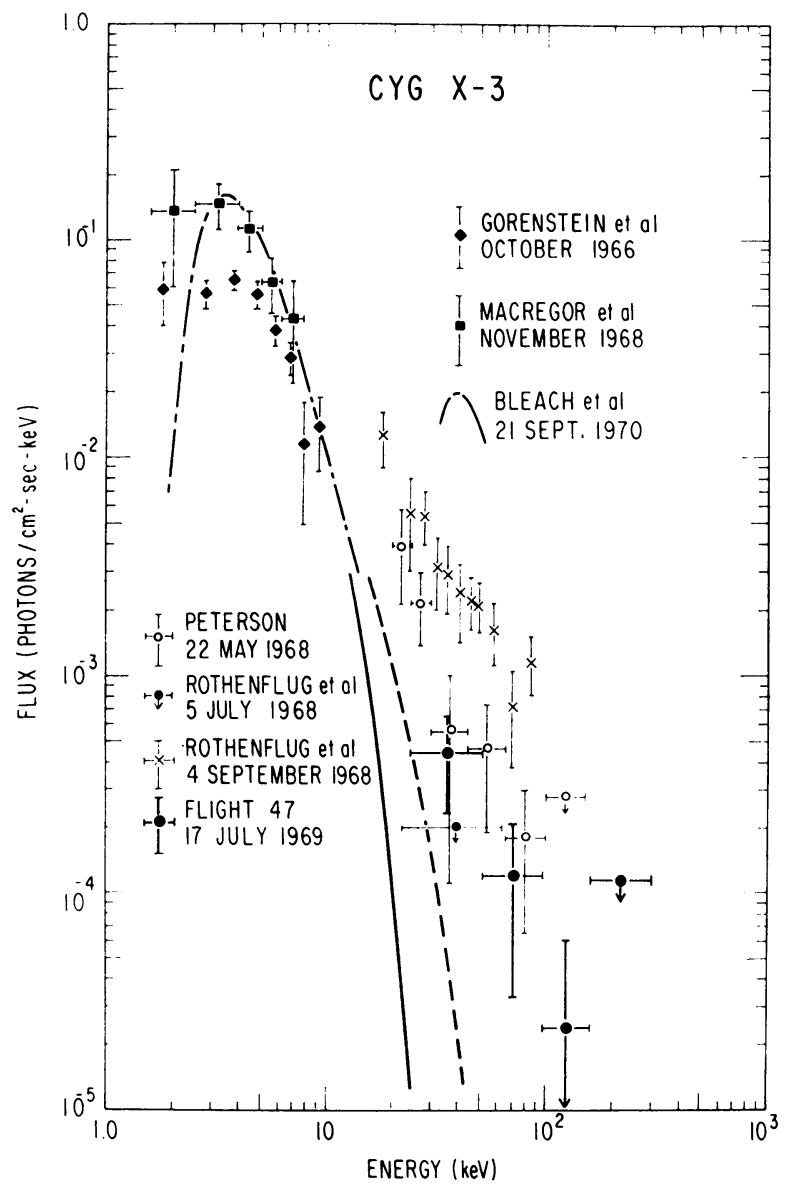

Fig. 11. CYG-3 has a remarkable similarity to CYG-1 as this collection of data (Matteson, 1971) shows. The flux at $100 \mathrm{keV}$ is sometimes that of CYG-1 with a factor of ten variations occurring at energies above $20 \mathrm{keV}$. The relative lack of $\mathrm{X}$-rays at energies below $4 \mathrm{keV}$ may be due to absoprtion in a column density of more than $10^{23}$ atoms $/ \mathrm{cm}^{2}$. Extrapolation of fits to the low energy spectrum indicated by the solid and broken lines cannot account for the high energy spectra.

time predicted from the optical observations. Furthermore, no evidence has been found for periodic fluctuations in the $160 \mathrm{~ms}$ to $1.2 \mathrm{~s}$ range by Agrawal et al. (1971b). Matteson (1971) has also searched for longer period variations and found none.

CYG-3, whose composite spectrum is shown in Figure 11 (Matteson, 1971), has many characteristics similar to CYG-1. At present no optical candidate exists for 
CYG-3, nor is there convincing evidence for binary modulation. This source does however exhibit absorption at lower energies (Bleach et al., 1971; Gorenstein et al., 1967).

E. NOVA-LIKE AND VARIABLE SOURCES

These sources, such as CEN-2 (Chodil et al., 1968), are characterized by a sudden increase in soft X-rays and a decay on a time scale typically that of galactic novae, 30 days.

In view of the re-determination of the position of CEN 2 (Francey, 1971) and the consequent re-evaluation of previous balloon observations, (Lewin et al., 1971b) it is not clear that these sources are observable in hard X-rays. It is clear that some sources, such as GX $1+4$ and GX 301-1 have appeared after not being seen in earlier surveys of the same region, and continue to be observed. The time behaviour of these sources in soft X-rays is presently unknown.

Some sources such as GX 304-1 have general variability. VEL-1, identified with 2U0900-40, may also be in this class. This object was scanned by the OSO-7 from December 17, 1971 to January 10, 1972. Observed time variations shown in Figure 12 are obtained from about four orbits per day of quick-look data. Each point represents an average of one hour of data for each available orbit. Despite clear evidence of variability, there is no obvious periodicity or pattern in these preliminary observations.

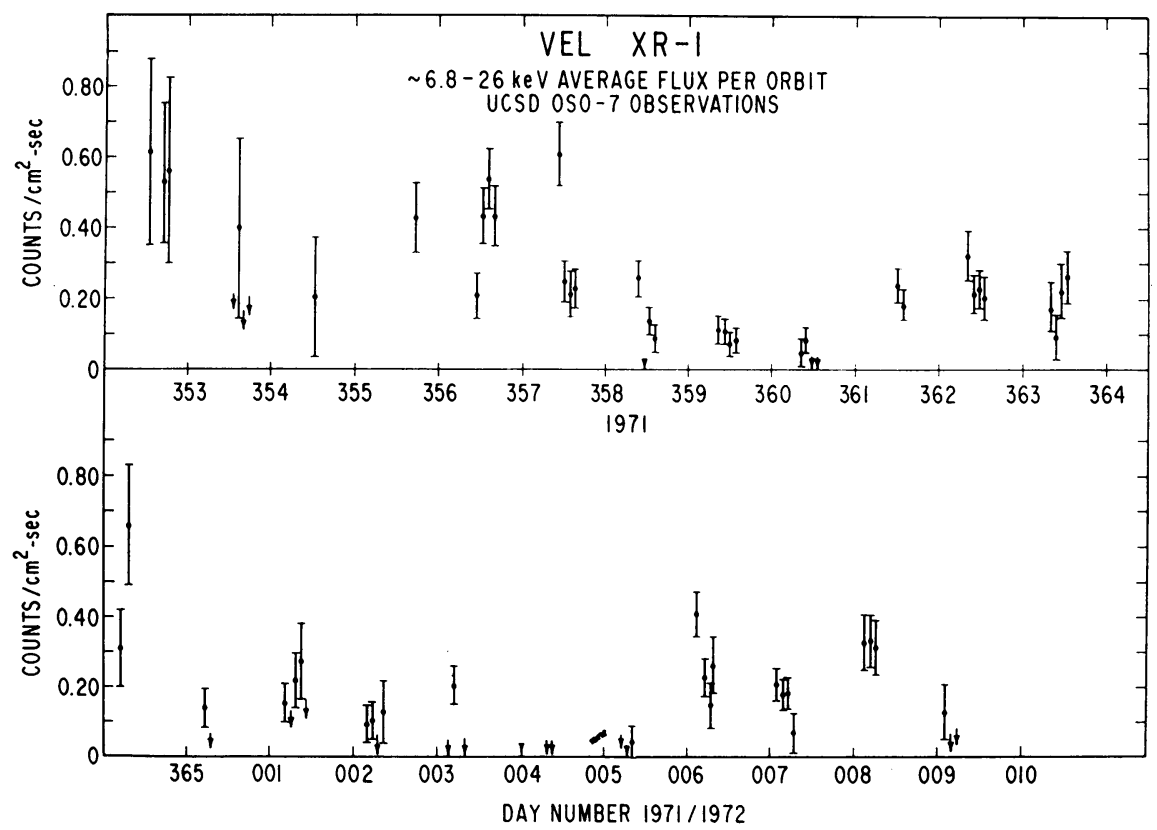

Fig. 12. Averages of the 7-26 keV X-ray flux from VEL-1 over selected one hour intervals during a two week period show considerable variability but no obvious periodicities

in the $\sim 1$ day to 10 day regime. 


\section{Extra Galactic Sources}

CEN A is the only extra-galactic source whose measured spectrum clearly extends to high energies. The total spectrum, taken from the work of Lampton et al. (1972) is shown in Figure 13, and is represented by a power law whose index is between -1.45 and -2.0 over the $1-180 \mathrm{keV}$ range. This object has been compared with other extragalactic emitters detected by UHURU (Kellogg et al., 1971).

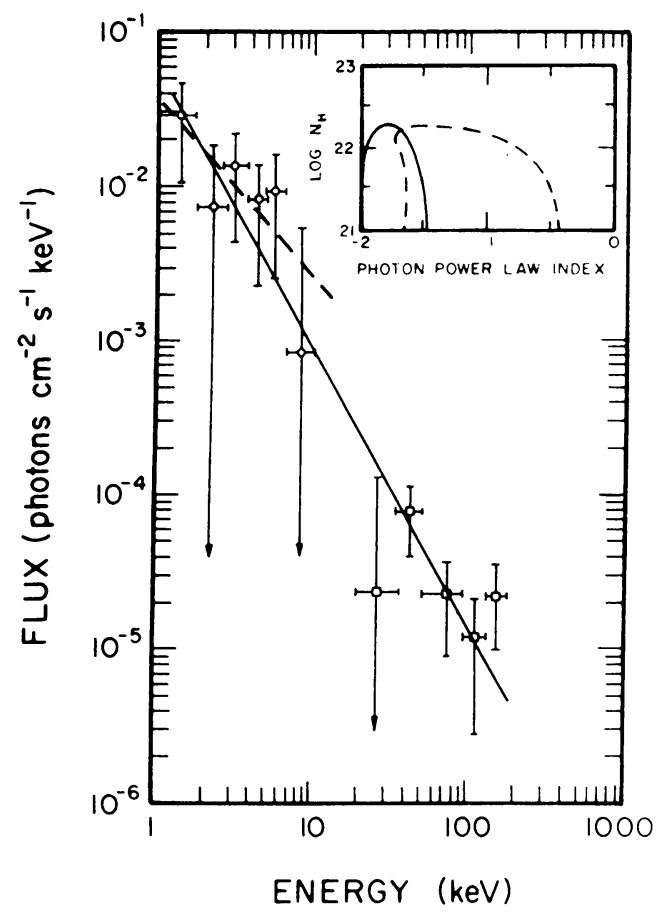

Fig. 13. Spectrum of NGC 5128 extending over the $1-180 \mathrm{keV}$ range. Errors are $\pm 1 \sigma$ statistical confidence. The broken line shows the best power-law fit to rocket data only, while the solid line is the best power-law fit to all data. The inset shows contours of constant probability for the fits. (Lampton et al., 1972).

Price et al. (1971) have detected the SMC to about $50 \mathrm{keV}$ and determined an energy spectrum with an index $\alpha=0.15 \pm 0.20$. This flux seems to disagree with an upper limit reported by Lewin et al. (1968b). Since the hard source noted by the UHURU (Leong et al., 1971) in the Small Magellanic Cloud is apparently compact and variable, these observations may be consistent.

Data from the Virgo region is usually reported as relevant to M87, even though telescope apertures do not separate M 87 from other objects in Virgo. Although Haymes (Fishman et al., 1970) has reported detectable fluxes on several occasions, other workers (Peterson, 1970; McClintock et al., 1969) have indicated substantially 
lower upper limits. Figure 14 shows a summary of recent measurements and upper limits taken from Laros et al. (1972), reporting recent observations by UCSD on extragalactic emitters. UCSD may have detected a flux from the Virgo region at the $2 \sigma$ confidence level. If all observations are assumed correct, a two component model is required, one of which produces a soft spectrum associated with the radio galaxy $M 87$, and the other a hard component due to at least one other compact and variable object.

The Andromeda Nebula (M31), 3C273, and the Large Magellanic Cloud have also been searched for hard X-ray emissions. The most recent upper limit obtained by

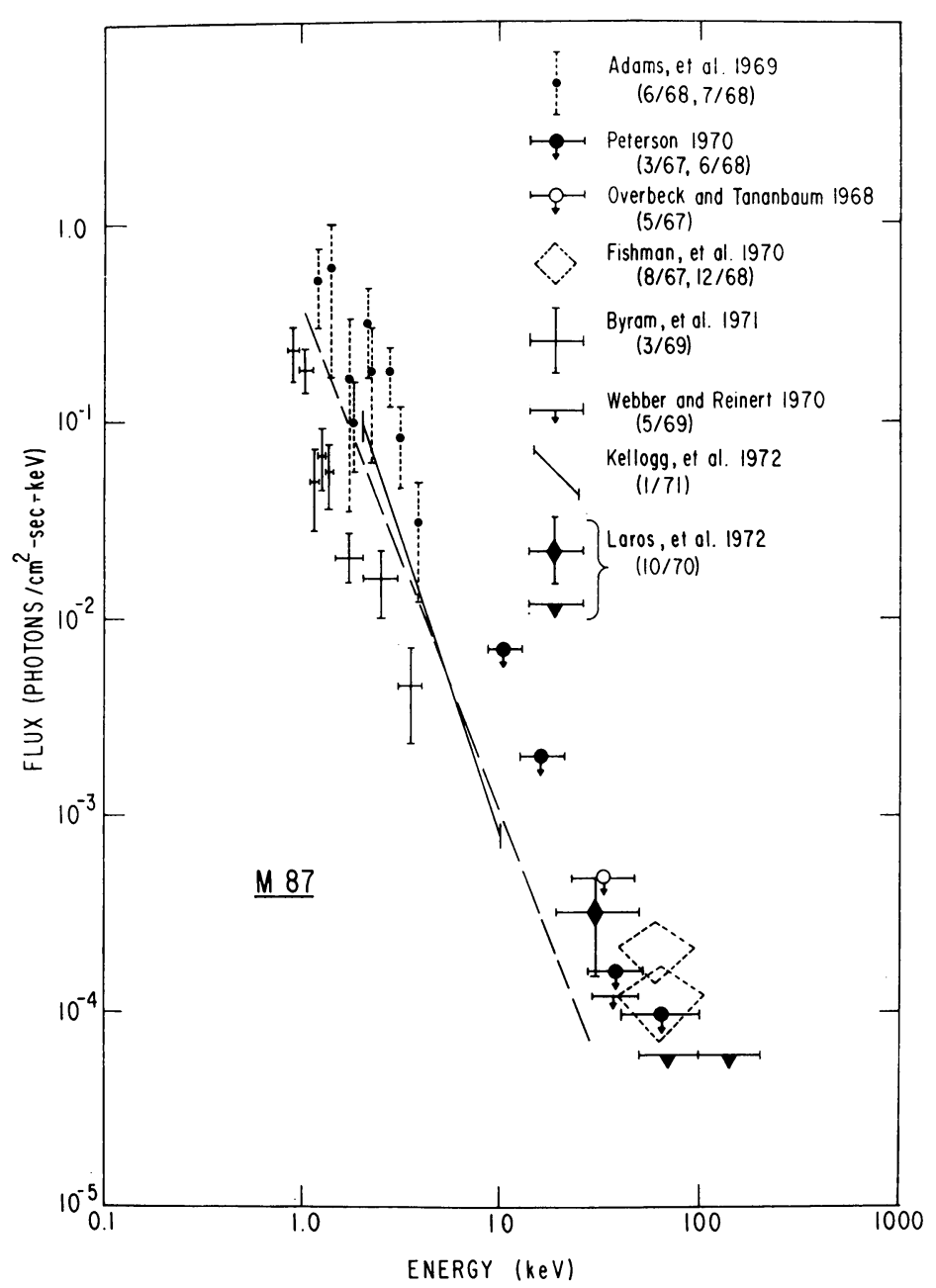

Fig. 14. Selected X-ray observations of M87 (Laros et al., 1972) showing observation dates. Mc Clintock et al. (1969) also has an upper limit in the 35-52 keV range of $1 \times 10^{-4}$ photons $\left(\mathrm{cm}^{2} \mathrm{~s} \mathrm{keV}\right)^{-1}$ at the $1 \sigma$ confidence level. A power law average of all the data in the $1-10 \mathrm{keV}$ (dashed line) is shown along with the single most sensitive measurement (solid line) and the extreme measurements which have been taken as evidence for variability. 




Fig. 15. X-ray observations of M31, including recent upper limits by UCSD. The positive result obtained by the UHURU satellite has yet to be confirmed by other experiments.

UCSD on M31 is shown in Figure 15. A comparable limit has also been obtained for 3C 273. A2 $\sigma$ upper limit of $\sim 6 \times 10^{-4}$ photons $\left(\mathrm{cm}^{2} \mathrm{~s} \mathrm{keV}\right)^{-1}$ over the $24-52 \mathrm{keV}$ range was also obtained by Matteson (1971) for CYG A.

\section{Conclusion}

This paper summarizes the present status of X-ray observations in the $20 \simeq 500 \mathrm{keV}$ range on galactic and extra-galactic sources. Because of limitations of sensitivity and observing time, results have not been as extensive as those in the range where the UHURU has produced a wealth of new discoveries. Hard X-ray emission is a certain indicator of non-thermal particle populations, and must, therefore, be associated with energetic processes in extreme astrophysical situations. In the immediate future, the OSO-7 will provide new results. By 1975 the HEAO-A will carry instruments to provide a further increment in sensitivity and observation time.

\section{Acknowledgements}

The author ackowledges useful discussions with various investigators regarding the contents of this paper. Dr Mel Ulmer in particular has provided extensive discussion 
and criticism of the manuscript. Many of my associates, Drs Baity, Laros, Matteson, Pelling, Ulmer and Wheaton have also contributed results which appear in this paper. This research was supported by NASA under Contracts NGR 05-005-003 and NAS 5-11080.

Note added in proof. Further analysis has discovered an $8.7 \pm 0.2 \mathrm{~d}$ periodicity for Vela X-1 X-rays.

\section{References}

Agrawal, P. C., Biswas, S., Gokhale, G. S., Iyengar, V. S., Kunte, P. K., Manchanda, R. K., and Sreekantan, B. V.: 1969, Proc. 11th Int. Conference on Cosmic Rays, Budapest, Hungary.

Agrawal, P. C., Biswas, S., Gokhale, G. S., Iyengar, V. S., Kunte, P. K., Manchanda, R. K., and Sreekantan, B. V.: 1971a, Proc. 12th Int. Conference on Cosmic Rays, Hobart (Australia), 1, 20.

Agrawal, P. C., Gokhale, G. S., Iyengar, V. S., Kunte, P. K., Manchanda, R. K., and Sreekantan, B. V.: 1971b, Proc. 12th Int. Conference on Cosmic Rays, Hobart (Australia), 1, 26.

Angel, J. R. P., Kestenbaum, H., and Novick, R.: 1971, Astrophys. J. Letters 169, L57.

Bleach, R. D., Boldt, E. A., Holt, S. S., Schwartz, D. A., and Serlemitsos, P. J.: 1972, Astrophys. J. 171, 51 .

Boldt, E., Doong, H., Serlemitsos, P., and Reigler, G. R.: 1968, Can. J. Phys. 46, S444.

Bolton, C. T.: 1972, Nature 235, 271.

Bradt, H., Burnett, B., Mayer, W., Rappaport, S., and Schnopper, H.: 1971, Nature 229, 96.

Chodil, G., Mark, H., Rodrigues, R., and Swift, C. D.: 1968, Astrophys. J. Letters 152, L45.

Fishman, G. J., Harnden, F. R., Jr., and Haymes, R. C.: 1970, in L. Gratton (ed.), 'Non-Solar X- and Gamma-Ray Astronomy', IAU Symp. 37, 116.

Francey, R. J.: 1971, Nature 229, 229.

Frost, K. J.: 1969, Astrophys. J. Letters 158, L159.

Giacconi, R., Kellogg, E., Gorenstein, P., Gursky, H., and Tananbaum, H.: 1971, Astrophys. J. Letters 165, L27.

Giacconi, R., Murray, S., Gursky, H., Kellogg, E., Schreier, E., and Tananbaum, H.: 1972, ASE2919, American Science \& Engineering, Cambridge, Mass. (submitted to Astrophys. J.).

Glass, I. S.: 1969, Astrophys. J. 157, 215.

Gorenstein, P., Giacconi, R., and Gursky, H.: 1967, Astrophys. J. Letters 150, L85.

Gorenstein, P., Kellogg, E. M., and Gursky, H.: 1970, Astrophys. J. 160, 199.

Harnden, F. R., Jr., Johnson, W. N., III, and Haymes, R. C.: 1972, Astrophys. J. Letters 172, L91.

Haymes, R. C. and Harnden, F. R., Jr.: 1970, Astrophys. J. 159, 1111.

Haymes, R. C., Harnden, F. R., Jr., Johnson, W. N., III, Prichard, H. M., and Bosch, H. E.: 1972, Astrophys. J. Letters 172, L47.

Hill, R. W., Burginyon, G., Grader, R. J., Palmieri, T. M., Seward, F. D., and Stoering, J. P.: 1972, Astrophys. J. 171, 519.

Hudson, H. S., Peterson, L. E., and Schwartz, D. A.: 1970, Astrophys. J. Letters 159, L51.

Jacobson, A S.: 1968, Ph. D. Thesis, UCSD.

Johnson, W. N., III., Harnden, F. R. Jr., and Haymes, R. C.: 1972, Astrophys. J. Letters 172, L1.

Kellogg, E., Gursky, H., Leong, C., Schreier, E., Tananbaum, H., and Giacconi, R.: 1971, Astrophys. J. Letters 165, L49.

Kinzer, R. L., Noggle, R. C., Seeman, N., and Shore, G. H.: 1971, Nature 229, 187.

Kraft, R. P. and Demoulin, M.: 1967, Astrophys. J. Letters 150, L183.

Kurfess, J. D.: 1971, Astrophys. J. Letters 168, L39.

Lampton, M., Bowyer, S., Welch, J., and Grasdalen, G.: 1971, Astrophys. J. Letters 164, L61.

Lampton, M., Margon, B., Bowyer, S., Mahoney, W., and Anderson, K.: 1972, Astrophys. J. Letters 171, L45.

Laros, J. G., Matteson, J. L., and Pelling, R. M.: 1972, UCSD (submitted for publication to Astrophys. J.)

Leong, C. Kellogg, E., Gursky, H., Tananbaum, H., and Giacconi, R.: 1971, Astrophys. J. Letters 170, L67.

Lewin, W. H. G., Clark, G. W., and Smith, W. B.: 1968a, Astrophys. J. Letters 152, L55. 
Lewin, W. H. G., Clark, G. W., and Smith, W. B.: 1968b, Nature 220, 249.

Lewin, W. H. G., McClintock, J. W., Ryckman, S. G., Glass, I. S., and Smith, W. B.: 1970a, Astrophys. J. Letters 162, L109.

Lewin, W. H. G., McClintock, J. E., and Smith, W. B.: 1970b, Astrophys. J. Letters 159, L193.

Lewin, W. H. G., Ricker, G. R., and McClintock, J. E.: 1971a, Astrophys. J. Letters 169, L17.

Lewin, W. H. G., McClintock, J. E., Ryckman, S. C., and Smith, W. B.: 1971b, Astrophys. J. Letters 166, L69.

Matteson, J. L.: 1971, 'An X-ray Survey of the Cygnus Region in the 20-300 keV Energy Range', Thesis, UCSD.

McClintock, J. E., Lewin, W. H. G., Sullivan, R. J., and Clark, G. W.: 1969, Nature 223, 162.

McClintock, J. E., Ricker, G. R., and Lewin, W. H. G.: 1971, Astrophys. J. Letters 166, L 73.

McClintock, J. E., Ricker, G. R., Ryckman, S. G., and Lewin, W. H. G.: 1972, Astrophys. J. Letters 173, L57.

Orwig, L. E., Chupp, E. L., and Forrest, D. J.: 1971, Nature 231, 171.

Overbeck, J. W. and Tananbaum, H. D.: 1968, Astrophys. J. 153, 899.

Pelling, R. M.: 1971, 'A Study of Simultaneous Optical and X-ray Observations of Scorpius X-1,' Thesis, UCSD.

Peterson, L. E.: 1970, in L. Gratton (ed.), 'Non-Solar X- and Gamma-Ray Astronomy', IAU Symp. $37,59$.

Peterson, L. E. and Jacobson, A. S.: 1970, Publ. Astron. Soc. Pac. 82, 412.

Peterson, L. E., Pelling, R. M., and Matteson, J. L.: 1971, UCSD-SP-71-04, SPARMO Symp., Seattle, Wash. (to be published in Space Sci. Rev.).

Price, R. E., Groves, D. J., Rodrigues, R. M., Seward, F. D., Swift, C. D., and Toor, A.: 1971, Astrophys. J. Letters 168, L7.

Riegler, G. R.: 1969, 'An X-Ray Survey of the Sky From Balloon Altitudes', Thesis, University of Maryland.

Schreier, E., Levinson, R., Gursky, H., Kellogg, E., Tananbaum, H., and Giacconi, R.: 1972, Astrophys. J. Letters 172, L79.

Schwartz, D. A.: 1970, Astrophys. J. 162, 439.

Schwartz, D. A., Hudson, H. S., and Peterson, L. E.: 1970, Astrophys. J. 162, 431.

Schwartz, D. A., Peterson, L. E., and Hudson, H. S.: 1971, Preprint X-661-71-445, Goddard Space Flight Center, Maryland (to be published in Astrophys. J.).

Terrell, N. J., Jr.: 1972, Astrophys. J. Letters 174, L35.

Toor, A., Seward, F. D., Cathey, L. R., and Kunkel, W. E.: 1970, Astrophys. J. 160, 209.

Webber, W. R. and Reinert, C. P.: 1970, Astrophys. J. 162, 883.

Webster, B. L. and Murdin, P.: 1972, Nature 235, 37. 